\title{
Decay of Charged Higgs boson in a scenario of SUSY breaking inspired neutrino mass
}

\author{
Gi-Chol Cho $^{a)}$, Satoru Kaneko ${ }^{a} * *$ and Aya Omote ${ }^{b)}$ \\ a) Department of Physics, Ochanomizu University, Tokyo 112-8610, Japan \\ b) Graduate School of Humanities and Sciences, Ochanomizu University, Tokyo, \\ 112-8610, Japan
}

\begin{abstract}
In some class of supersymmetric models, small neutrino mass is given as a consequence of the supersymmetry (SUSY) breaking. Phenomenologically interesting features of this scenario are as follows: (i) the right-handed sneutrino mass could be as low as $\mathrm{TeV}$ scale due to the GiudiceMasiero mechanism, and (ii) a scalar trilinear interaction of Higgs-slepton-(right-handed) sneutrino could be sizable without suppression by the small neutrino Yukawa coupling. We study some phenomenological aspects of this scenario focusing on the scalar trilinear interaction. We show that the 1-loop correction by sneutrino exchange to the lightest Higgs boson mass destructively interferes with top-stop contributions in the minimal SUSY Standard Model. We find that a decay of charged Higgs boson into sneutrino and charged slepton is sizably enhanced and hence it gives rise to a distinctive signal at future collider experiments in some parameter space.
\end{abstract}

* present address: AHEP Group, Instituto de Física Corpuscular - C.S.I.C./Universitat de València, Edifici Instituts d'Investigacio, Apt. 22085, E-46071 València, Spain 


\section{Introduction}

Smallness of neutrino mass is one of the important clues to physics beyond the Standard Model (SM). An attractive explanation on the origin of small neutrino mass is the seesaw mechanism [1]. In the seesaw mechanism, a heavy right-handed neutrino is introduced and it couples to $\mathrm{SU}(2)_{L}$ doublet neutrino and Higgs boson through the Yukawa coupling $Y_{\nu}$. After diagonalizing the neutrino mass matrix, a smaller mass eigenvalue of neutrino, $m_{\nu}$, is given by

$$
m_{\nu} \simeq\left(Y_{\nu} v\right)^{2} / m_{N}
$$

where $m_{N}$ and $v$ are the mass of right-handed neutrino and the vacuum expectation value (v.e.v.) of the Higgs boson, respectively. If the Yukawa coupling $Y_{\nu}$ is of order unity, the right-handed neutrino should be heavy enough, say, $m_{N} \sim 10^{11} \mathrm{GeV} /\left(m_{\nu} / 1 \mathrm{eV}\right)$, to be consistent with results of neutrino experiments. Then one may complain the large hierarchy between the scale of the seesaw mechanism $\left(m_{N}\right)$ and the electroweak scale $(v)$. Furthermore it is hopeless to confirm the seesaw mechanism through searching for the right-handed neutrino at collider experiments (a trial to test the seesaw mechanism with hypothetical outcome of future experiments is proposed in ref.[3]). Thus it may be worth considering a possibility to lower the scale of seesaw mechanism (scale of righthanded neutrino) as low as testable at collider experiments, say, $O(100 \mathrm{GeV}-1 \mathrm{TeV})$, or alternative to the seesaw mechanism from a phenomenological point of view.

It has been argued possibilities to explain the small neutrino mass as a consequence of supersymmetry (SUSY) breaking in refs. [4, 5, 6]. Some phenomenologically viable points of this class of models are (i) light ( $\mathrm{TeV}$ scale) right-handed sneutrino due to the Giudice-Masiero mechanism [7] and (ii) enhancement of scalar trilinear interaction among the right-handed sneutrino, left-handed slepton and Higgs bosons. Both (i) and (ii) can be, for example, realized as follows. Let us first introduce a chiral superfield $X$ which is a SM gauge singlet but charged under a certain global symmetry. This global symmetry may allow non-renormalizable operators such as

$$
\frac{X X^{\dagger}}{M_{P}^{2}} N^{\dagger} N
$$

and

$$
\frac{X}{M_{P}} L H_{u} N
$$

where dimensionless couplings with $O(1)$ magnitude are suppressed. In (21) and (3), $L$ and $N$ denote the left-handed lepton and right-handed neutrino superfields, respectively. 
The Higgs superfield with the hypercharge $Y=1 / 2$ is represented by $H_{u}$, and $M_{P}$ is the reduced Planck mass. Suppose that the $F$-component of the $X$ field develops a v.e.v. $\langle F\rangle \sim m_{3 / 2} M_{P}$ due to the SUSY breaking, where $m_{3 / 2}$ is the gravitino mass. Then the $D$-component of (2) leads to the right-handed sneutrino mass as

$$
\left(\frac{X X^{\dagger}}{M_{P}^{2}} N^{\dagger} N\right)_{D} \rightarrow m_{\widetilde{\nu}_{R}}^{2} \widetilde{\nu}_{R}^{*} \widetilde{\nu}_{R},
$$

while the $F$-component of (3) gives the scalar trilinear interaction

$$
\left(\frac{X}{M_{P}} L H_{u} N\right)_{F} \rightarrow A_{\nu} \widetilde{\ell} H_{u} \widetilde{\nu}_{R}
$$

Note that both $m_{\widetilde{\nu}_{R}}$ and $A_{\nu}$ are of order the gravitino mass $\sim O(\mathrm{TeV})$. Moreover the scalar trilinear interaction is not suppressed if a dimensionless coupling in (3) is of order unity.

In the minial SUSY SM (MSSM), the SUSY breaking scalar trilinear interactions of squark or sleptons are parametrized by $A_{f} Y_{f}$, where $A_{f}$ and $Y_{f}$ are the scalar trilinear coupling and the Yukawa coupling for flavor $f$, respectively. The scalar three-point vertices are, therefore, suppressed by small Yukawa couplings for the first two generations of squarks and sleptons. In the models of refs. [4, 5, 6], however, the scalar trilinear interaction of the right-handed sneutrino is not suppressed by the neutrino Yukawa coupling, as mentioned above.

A few comments on this class of models are in order. In a series of non-renormalizable operators ((2) , (3) , etc), there are lepton-number violating operators in general. If such operators are forbidden by an appropriate discrete symmetry, the seesaw mechanism does not work and the Dirac neutrino mass should be given by (3) with the $A$-component v.e.v. $\langle A\rangle$ of the $X$-field. Then, to satisfy the experimental limit, a relation $\langle A\rangle \ll \sqrt{\langle F\rangle}$ must hold. On the other hand, if the lepton-number violating operators are allowed, the Majorana mass term of the right-handed neutrino and the SUSY breaking $B$-term of right-handed sneutrino appear at the same order of magnitude. This $B$-term contributes to the smaller neutrino mass through the radiative correction and, therefore, should be highly suppressed. Further discussions on these constraints can be found in refs. [5, 8].

In this paper, we investigate phenomenological consequences of a scenario of $\mathrm{TeV}$ scale right-handed sneutrino inspired by supersymmetric models in refs. [4, 5, 6], focusing on the unsuppressed coupling $A_{\nu}$. We first study the 1-loop corrections to the lightest Higgs boson mass through the sneutrino exchange which is proportional to some powers of $A_{\nu}$. We show that the sneutrino contribution to the lightest Higgs boson mass destructively interferes with the MSSM contribution. We next study decay processes 
of charged Higgs boson [4]. Owing to $A_{\nu}$, the decay of charged Higgs boson into the sneutrino and selectron could be enhanced as compared to the MSSM. We find that, in some parameter space, the branching ratio of this decay mode can be as large as $10 \%$, and it may be detectable at future linear collider experiments. In our study, we neglect the generation mixing in both the left- and right-handed sneutrinos for simplicity. Although this scenario has a possibility if the neutrino is Majorana or Dirac, our study is available in both cases if the SUSY breaking $B$-term of sneutrino in the Majorana case is assumed to be small enough so that, in addition to suppress the 1-loop correction to the mass of lighter neutrino, the sneutrino mass matrix has common structure in both cases.

\section{Mass and Interactions}

We first review the sneutrino masses and interactions to fix our notation. When the SUSY breaking $B$-term of sneutrino is neglected, the mass matrix of sneutrinos in a basis of $\left(\widetilde{\nu}_{L}, \widetilde{\nu}_{R}\right)$ is given by

$$
\begin{aligned}
M_{\tilde{\nu}}^{2} & =\left(\begin{array}{cc}
m_{\widetilde{\nu}_{L}}^{2} & A_{\nu} v \sin \beta \\
A_{\nu} v \sin \beta & m_{\widetilde{\nu}_{R}}^{2}
\end{array}\right), \\
m_{\widetilde{\nu}_{L}}^{2} & =m_{L}^{2}+\frac{1}{2} \cos 2 \beta m_{Z}^{2},
\end{aligned}
$$

where $m_{L}$ is the soft scalar mass for the $\mathrm{SU}(2)_{L}$ doublet slepton while $m_{\widetilde{\nu}_{R}}$ is for the right-handed sneutrino. The angle $\beta$ is defined as $\tan \beta \equiv v_{u} / v_{d}$, where $v_{u}$ and $v_{d}$ are v.e.v. of the Higgs bosons with $Y=1 / 2$ and $-1 / 2$, respectively. A parameter $v$ is

normalized as $v \equiv \sqrt{v_{u}^{2}+v_{d}^{2}} \approx 246 \mathrm{GeV}$. The mass matrix (6) can be diagonalized using an unitary matrix $U_{\widetilde{\nu}}$ :

$$
\left(U_{\widetilde{\nu}}\right)^{\dagger} M_{\widetilde{\nu}}^{2} U_{\widetilde{\nu}}=\operatorname{diag}\left(m_{\widetilde{\nu_{1}}}, m_{\widetilde{\nu_{2}}}\right), \quad\left(m_{\widetilde{\nu}_{1}}<m_{\widetilde{\nu_{2}}}\right) .
$$

In the MSSM, the sneutrino mass is given by (17). Note that $m_{\widetilde{\nu}_{L}}^{2}$ (17) satisfies the following relation with the mass of left-handed selectron $\widetilde{e}_{L}$ due to the $\mathrm{SU}(2)_{L}$ symmetry:

$$
m_{\tilde{e}_{L}}^{2}-m_{\tilde{\nu}_{L}}^{2}=\left(-1+s_{W}^{2}\right) m_{Z}^{2} \cos 2 \beta .
$$

Since $\cos 2 \beta<1$ for $\tan \beta>1$, the mass of sneutrino in the MSSM is always smaller than the selectron mass when $\tan \beta>1$. On the other hand, the lighter sneutrino mass (8) is independent of the selectron mass and can be much lighter than the sneutrino in the MSSM. 

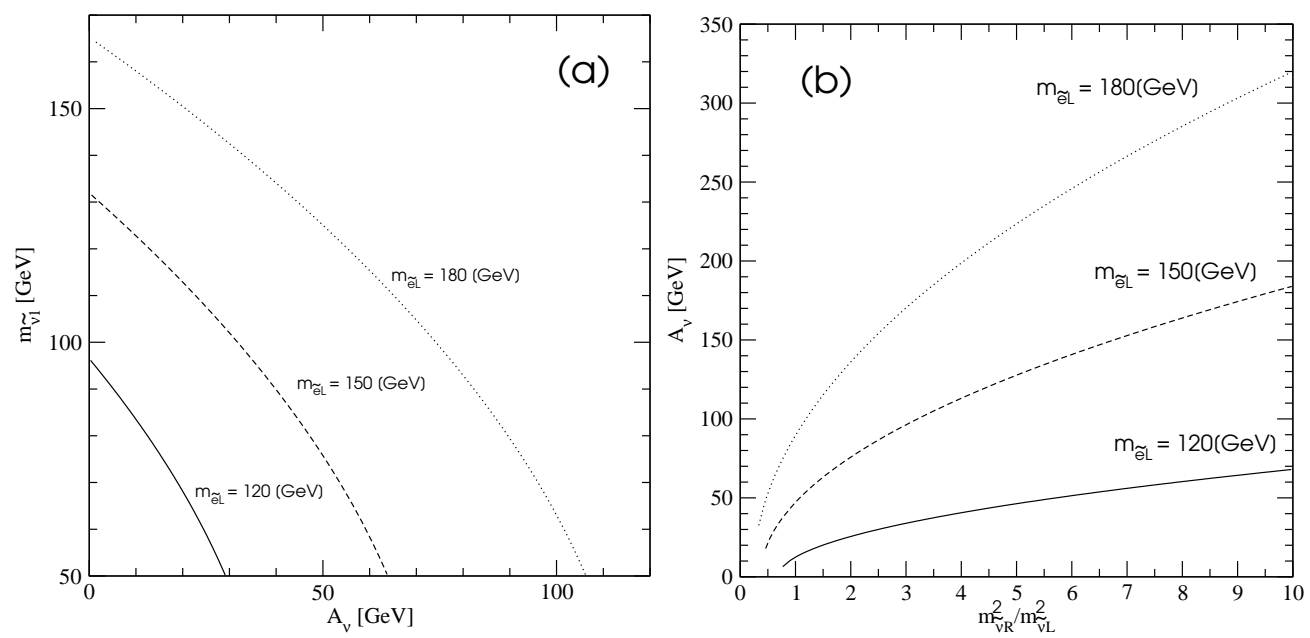

Figure 1: (a): The lighter sneutrino mass $m_{\widetilde{\nu}_{1}}$ as a function of $A_{\nu}$ for $\tan \beta=3$. Three lines correspond to $m_{\widetilde{e}_{L}}=120 \mathrm{GeV}$ (solid), $150 \mathrm{GeV}$ (dashed) and $180 \mathrm{GeV}$ (dotted). The results are obtained by taking $m_{\widetilde{\nu}_{L}}^{2}=m_{\widetilde{\nu}_{R}}^{2}$. (b): The coupling $A_{\nu}$ as a function of a ratio $m_{\widetilde{\nu}_{R}}^{2} / m_{\widetilde{\nu}_{L}}^{2}$ for $\tan \beta=3$.

In Fig. 1(a), we show the lighter sneutrino mass $m_{\widetilde{\nu}_{1}}$ as a function of $A_{\nu}$ for $\tan \beta=$ 3. Three lines correspond to $m_{\widetilde{e}_{L}}=120 \mathrm{GeV}$ (solid), $150 \mathrm{GeV}$ (dashed) and $180 \mathrm{GeV}$ (dotted). For the right-handed sneutrino mass, we take $m_{\widetilde{\nu}_{R}}=m_{\widetilde{\nu}_{L}}$ for convenience. Note that the mass $m_{\widetilde{\nu}_{1}}$ at $A_{\nu}=0$ corresponds to that in the MSSM. The figure tells us that the large left-right mixing of sneutrino which is induced by large $A_{\nu}$, makes a sneutrino much lighter than that in the MSSM.

Fig. 1(b) shows the trilinear coupling $A_{\nu}$ as a function of $m_{\widetilde{\nu}_{R}}^{2} / m_{\widetilde{\nu}_{L}}^{2}$. Three lines correspond to different values of the selectron mass as Fig. 1(a). The lighter sneutrino mass $m_{\widetilde{\nu}_{1}}$ is fixed at $80 \mathrm{GeV}$. It can be seen from the figure that the coupling $A_{\nu}$ increases when $m_{\widetilde{\nu}_{R}}^{2} / m_{\widetilde{\nu}_{L}}^{2}$ is larger than one.

Next we summarize the interaction Lagrangian of sneutrino, slepton and Higgs bosons. For simplicity we take a limit of large pseudo scalar mass $m_{A}$. Then the lightest Higgs boson $h$ can be approximately identified with the SM Higgs. The interaction Lagrangians of sneutrino-sneutrino-lightest Higgs boson $\left(\widetilde{\nu}_{i}-\widetilde{\nu}_{j}-h\right)$ and sneutrinoslepton-charged Higgs boson $\left(\widetilde{\nu}_{i}-\widetilde{\ell}-H^{-}\right)$are then given as follows:

- $\widetilde{\nu}_{i}-\widetilde{\nu}_{j}-h$ interaction:

$$
\mathcal{L}=A_{\nu} \sum_{i, j}\left(U_{\widetilde{\nu}}\right)_{1 i}^{*}\left(U_{\widetilde{\nu}}\right)_{2 j} \widetilde{\nu}_{i}^{*} \widetilde{\nu}_{j} h+\text { h.c. } \quad(i, j=1,2)
$$



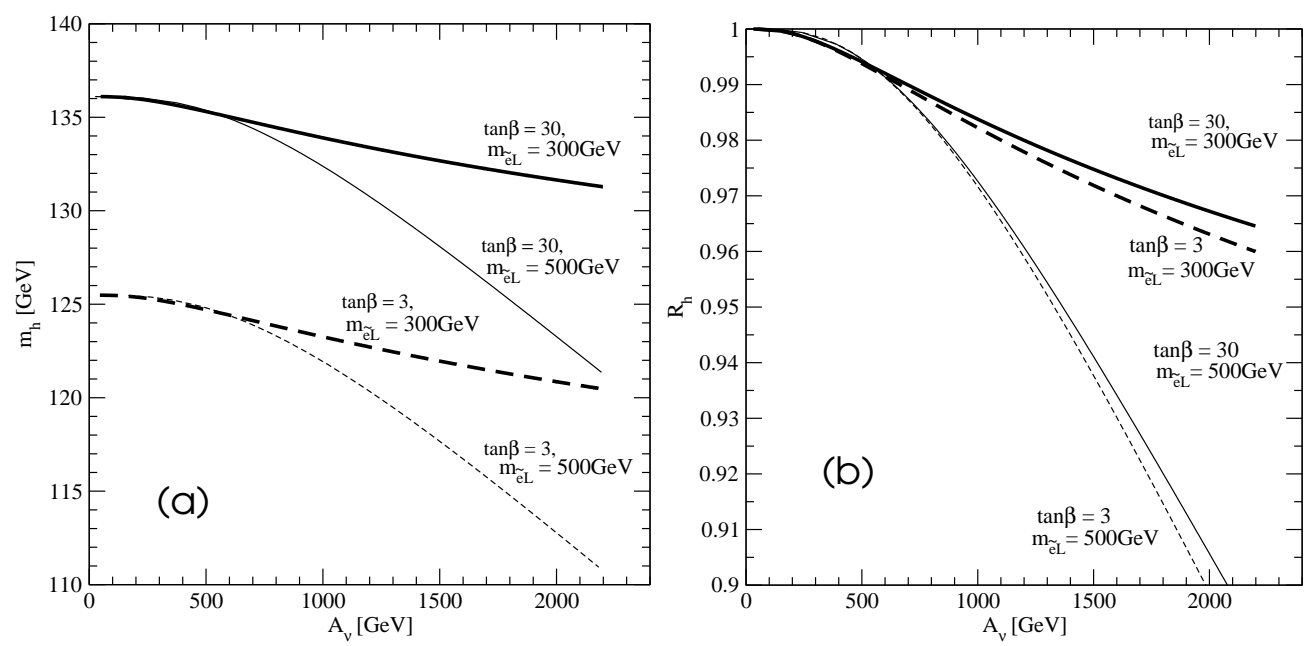

Figure 2: (a) The lightest Higgs boson mass $m_{h}$ as a function of $A_{\nu}$. Each line correspond to combinations of $\tan \beta=3,30$ and $m_{\widetilde{e}_{L}}=300,500 \mathrm{GeV}$ as indicated. The 1-loop correction from the top-stop loop is evaluated following ref. 13. using the stop mass $m_{\tilde{t}}=1 \mathrm{TeV}$. The Higgs mass $m_{h}$ at $A_{\nu}=0$ corresponds to the MSSM prediction. (b) The ratio $R_{h}$ defined in eq.(17) as a function of $A_{\nu}$.

- $\widetilde{\nu}_{i}-\widetilde{\ell}-H^{-}$interaction:

$$
\begin{aligned}
\mathcal{L} & =g^{\widetilde{\nu}_{i} \tilde{\ell} H^{-}} \widetilde{\nu}_{i}^{*} \widetilde{\ell} H^{+}+\text {h.c. }, \\
g^{\widetilde{\nu_{i}} \widetilde{\ell} H^{-}} & =A_{\nu} \cos \beta\left(U_{\widetilde{\nu}}\right)_{2 i}^{*}-\frac{g}{\sqrt{2}} m_{W} \sin 2 \beta\left(U_{\widetilde{\nu}}\right)_{1 i}^{*} \quad(i=1,2) .
\end{aligned}
$$

\section{Sneutrino contribution to the lightest Higgs boson mass}

It is known that the lightest Higgs boson mass $m_{h}$ receives large 1-loop corrections mainly from the top quark and the stop exchanging diagram [9, 10, 11]. In the scenario of $\mathrm{TeV}$ scale $\widetilde{\nu}_{R}$ with sizable $A_{\nu}$, the $\widetilde{\nu}_{L}-\widetilde{\nu}_{R}-h$ interaction (10) could give a new contribution to the lightest Higgs boson mass at 1-loop level. Using the renormalization group method used in ref. [10, we evaluate the sneutrino contribution to $m_{h}$.

Let us take the large limit of the SUSY breaking mass scale $m_{\mathrm{SUSY}}$ so that physics below $m_{\text {SUSY }}$ is described by the Standard Model. Then the lightest Higgs boson mass $m_{h}$ is simply parametrized by

$$
m_{h}^{2}=\lambda v^{2}
$$

where $\lambda$ is a quartic coupling in the Higgs potential. Note that the quartic coupling at the tree level, $\lambda_{\text {tree}}$, satisfies the SUSY relation

$$
\lambda_{\text {tree }}=\frac{1}{4}\left(g_{Y}^{2}+g^{2}\right) \cos ^{2} 2 \beta,
$$


where $g_{Y}$ and $g$ are the $\mathrm{U}(1)_{Y}$ and $\mathrm{SU}(2)_{L}$ gauge couplings, respectively. The radiative corrections to the quartic coupling $\lambda$ in the MSSM can be found in, for example, ref. [10]. In the scenario of large $A_{\nu}$, the interaction (10) gives rise to the sneutrino exchanging box diagram as the 1-loop correction to the quartic coupling $\lambda$. The sneutrino contribution, $\lambda_{\tilde{\nu}}$, can be evaluated as

$$
\lambda_{\widetilde{\nu}}=-\frac{A_{\nu}^{4}}{4(4 \pi)^{2}} \sum_{i, j, k, l=1}^{2}\left|U_{1 i}^{\widetilde{\nu}}\right|^{2}\left|U_{2 j}^{\widetilde{\nu}}\right|^{2}\left|U_{1 k}^{\widetilde{\nu}}\right|^{2}\left|U_{2 l}^{\widetilde{\nu}}\right|^{2} D_{0}\left(m_{\widetilde{\nu_{i}}}, m_{\widetilde{\nu_{j}}}, m_{\widetilde{\nu_{k}}}, m_{\widetilde{\nu_{l}}}\right),
$$

where the 1-loop scalar function $D_{0}$ is given by

$$
\begin{aligned}
D_{0}\left(m_{1}, m_{2}, m_{3}, m_{4}\right)= & \frac{1}{\left(m_{1}^{2}-m_{2}^{2}\right)\left(m_{3}^{2}-m_{4}^{2}\right)}\left[\frac{m_{1}^{2}+m_{3}^{2}}{m_{1}^{2}-m_{3}^{2}} \ln \frac{m_{3}}{m_{1}}+\frac{m_{2}^{2}+m_{4}^{2}}{m_{2}^{2}-m_{4}^{2}} \ln \frac{m_{4}}{m_{2}}\right. \\
& \left.-\frac{m_{1}^{2}+m_{4}^{2}}{m_{1}^{2}-m_{4}^{2}} \ln \frac{m_{4}}{m_{1}}-\frac{m_{2}^{2}+m_{3}^{2}}{m_{2}^{2}-m_{3}^{2}} \ln \frac{m_{3}}{m_{2}}\right] .
\end{aligned}
$$

In Fig. 2(a), we depict the $A_{\nu}$ dependence of the lightest Higgs boson mass $m_{h}$. We also compare, in Fig. 2(b), a ratio of the Higgs boson mass in our scenario and in the MSSM which is defined as

$$
R_{h} \equiv \frac{m_{h}}{m_{h}(\mathrm{MSSM})} .
$$

In eq.(17), $m_{h}$ and $m_{h}(\mathrm{MSSM})$ are the lightest Higgs boson mass in our scenario and the Higgs mass in the MSSM, respectively. In the figures, the 1-loop corrections in the MSSM are estimated following ref. [13] with the stop mass $m_{\widetilde{t}}=1 \mathrm{TeV}$. Solid and dotted lines denote $m_{\widetilde{e}_{L}}=300 \mathrm{GeV}$ and $500 \mathrm{GeV}$, respectively. Thin and thick lines are $\tan \beta=3$ and 50 as indicated in the figures. Note that, in both figures, $m_{h}$ and $R_{h}$ at $A_{\nu}=0$ correspond to the MSSM prediction. It is easy to see that both $m_{h}$ and $R_{h}$ decrease when $A_{\nu}$ increases. This means that the sneutrino contribution to $m_{h}$ interferes with the MSSM contributions destructively. For example, in a limit where two sneutrino masses are equal ( $\left.m_{\mathrm{SUSY}}\right)$, the quartic coupling $\lambda_{\nu}$ is given as

$$
\lambda_{\nu} \simeq-\frac{1}{6} \frac{1}{(4 \pi)^{2}}\left(\frac{A_{\nu}}{m_{\mathrm{SUSY}}}\right)^{4}<0 .
$$

The minus sign in r.h.s. of (18) is the origin that $m_{h}$ is lowered via the sneutrino contribution. Fig. 2(b) shows that the negative contribution to $m_{h}$ from the sneutrino diagram is less than $5 \%$ for $A_{\nu} \lesssim 1 \mathrm{TeV}$.

\section{Decay of charged Higgs boson}

Next we examine a decay $H^{-} \rightarrow \widetilde{\nu}+\widetilde{\ell}$, where $H^{-}$stands for a charged Higgs boson. In particular, a case of $\widetilde{\ell}=\widetilde{e}$ could be a distinctive process of our scenario because that such 
process is strongly suppressed in the MSSM due to the electron Yukawa coupling. So, we consider only the case of $\tilde{\ell}=\widetilde{e}$ in the following study. In the MSSM, it is known that, for $m_{H^{-}} \gtrsim 200 \mathrm{GeV}, H^{-}$dominantly decays into the top and bottom quarks owing to the sizable Yukawa couplings (for a review of various decay channels of the charged Higgs boson in the supersymmetric models, see ref. [12]). The $\tau+\nu_{\tau}$ mode is subdominant for large $\tan \beta(\gtrsim 10)$ due to the tau-Yukawa coupling. On the other hand, when $A_{\nu}$ is sizable, it is expected that the decay mode $H^{-} \rightarrow \widetilde{\nu}_{1}+\widetilde{e}$ is much enhanced in small $\tan \beta$ region because that the decay vertex is proportional to $A_{\nu} \cos \beta$ (12). The decay width of $H^{-} \rightarrow \widetilde{\nu}_{1}+\widetilde{e}$ is given as follows:

$$
\Gamma\left(H^{-} \rightarrow \widetilde{\nu}_{1}+\widetilde{e}\right)=\frac{1}{16 \pi m_{H^{-}}}\left|g^{\widetilde{\nu}_{1} e H^{-}}\right|^{2} \kappa^{1 / 2}\left(1,\left(\frac{m_{\widetilde{\nu}_{1}}}{m_{H^{-}}}\right)^{2},\left(\frac{m_{\widetilde{e}}}{m_{H-}}\right)^{2}\right),
$$

where a function $\kappa$ is defined by

$$
\kappa(a, b, c) \equiv a^{2}+b^{2}+c^{2}-2 a b-2 a c-2 b c .
$$

In Fig. 3, we show branching ratios of some decay modes of the charged Higgs boson with $m_{H^{-}}=350 \mathrm{GeV}$ as functions of $\tan \beta$. We assume that squarks are heavy enough so that the decay modes into squarks are kinematically forbidden. Heavy squarks are also favored to make the lightest Higgs boson heavy through the radiative corrections, against for the negative contribution to $m_{h}$ from the sneutrino exchanging diagrams. The sneutrino and selectron masses are chosen as $m_{\widetilde{\nu}_{1}}=50 \mathrm{GeV}$ and $m_{\widetilde{e}_{L}}=200 \mathrm{GeV}$, respectively. The trilinear coupling of right-handed sneutrino $A_{\nu}$ is fixed at $500 \mathrm{GeV}$. Then the heavier sneutrino mass $\left(m_{\widetilde{\nu}_{2}}\right)$ is about $700 \mathrm{GeV}$. As already mentioned, we assumed the flavor universality of $A_{\nu}$, so the branching ratio of decay into the sneutrino and smuon, or stau, is same with the selectron mode shown in the figure. As an example, the branching ratio of decay into charginos $\left(\tilde{\chi}_{i}^{-}, i=1,2\right)$ and neutralinos $\left(\widetilde{\chi}_{j}^{0}, j=1,4\right)$ is examined for $m_{\widetilde{\chi}_{1}^{-}}=150 \mathrm{GeV}$ with $M_{2} / \mu=5$ in Fig. 33(a) and $M_{2} / \mu=1$ in Fig. 3(b), where $M_{2}$ and $\mu$ stand for the $\mathrm{SU}(2)_{L}$ gaugino mass and the higgsino mass, respectively. The $\mathrm{U}(1)_{Y}$ gaugino mass $M_{1}$ is obtained using the GUT relation, $M_{1} / \alpha_{Y}=(5 / 3)\left(M_{2} / \alpha_{2}\right)$, where $\alpha_{i}(i=Y, 2)$ are given as $\alpha_{i}=g_{i}^{2} /(4 \pi)$. Then the mass of lightest neutralino is given as $m_{\widetilde{\chi}_{1}^{0}} \sim 142 \mathrm{GeV}$ in Fig. 3(a) and $93 \mathrm{GeV}$ in Fig. 3(b). The ratio $M_{2} / \mu$ determines the properties of the lighter chargino and the lightest neutralino. When $M_{2} / \mu \ll 1$ the lighter chargino is mostly the $\mathrm{SU}(2)_{L}$ gaugino while the relation $M_{2} / \mu \gg 1$ corresponds to the higgsino dominant case. For $M_{2} / \mu=5$, both the lighter chargino and the lightest neutralino are higgsino dominant, so that the decay $H^{-} \rightarrow \widetilde{\chi}_{1}^{-}+\widetilde{\chi}_{1}^{0}$ is highly suppressed because there is no Higgs-higgsino-higgsino coupling. This explains the difference of $\operatorname{Br}\left(H^{-} \rightarrow \widetilde{\chi}_{1}^{-}+\widetilde{\chi}_{1}^{0}\right)$ between Figs. 33(a) and (b). 
It can be seen from Fig. 3 that the branching ratio of $H^{-} \rightarrow \widetilde{\nu}+\widetilde{\ell}$ mode could be as large as $10 \%$ for small $\tan \beta(\lesssim 7)$. In the MSSM, the charged Higgs boson can decay into $\widetilde{\nu}_{L}$ and $\widetilde{e}_{R}$. For comparison, we fix the mass of $\widetilde{e}_{R}$ as $m_{\widetilde{e}_{R}}=m_{\widetilde{e}_{L}}=200 \mathrm{GeV}$. Then the decay mode $H^{-} \rightarrow \widetilde{\nu}_{L}+\widetilde{e}_{R}$ is kinematically forbidden because the sneutrino $\widetilde{\nu}_{L}$ cannot be much lighter than $\widetilde{e}_{L}$ due to the $\mathrm{SU}(2)_{L}$ relation (9) (note that $m_{\widetilde{e}_{R}}=m_{\widetilde{e}_{L}}=200 \mathrm{GeV}$ ). Therefore, if the charged Higgs boson mass does not differ so much from the masses of charged sleptons, the decay $H^{-} \rightarrow \widetilde{\nu}_{L}+\widetilde{e}_{R}$ in the MSSM is strongly suppressed.

Next we study a signal of the decay $H^{-} \rightarrow \widetilde{\nu}_{1}+\widetilde{e}_{L}$ in some detail. For our choice of the inputs used in Fig. 3, the selectron $\widetilde{e}_{L}$ dominantly decays into the lightest neutralino and an electron, $\widetilde{e}_{L} \rightarrow \widetilde{\chi}_{1}^{0}+e$. Then, since the branching ratio of the $\widetilde{\nu}_{1}+\widetilde{e}_{L}$ mode is roughly $10 \%$ for small $\tan \beta$ region, a probability which we find an electron from this decay mode can be estimated as $\operatorname{Br}\left(H^{-} \rightarrow \widetilde{\nu}_{1}+\widetilde{e}_{L}\right) \times \operatorname{Br}\left(\widetilde{e}_{L} \rightarrow e+\widetilde{\chi}_{1}^{0}\right) \simeq 10 \%$. The electron is also coming out from the $W$ boson of the decay $H^{-} \rightarrow W+h$, and the chargino of the decay $H^{-} \rightarrow \widetilde{\chi}^{-}+\widetilde{\chi}^{0}$. From Fig. 3 we find that $\operatorname{Br}\left(H^{-} \rightarrow W+h\right) \lesssim 3 \%$ and the leptonic decay of the $W$ boson is known as $\operatorname{Br}(W \rightarrow \nu+e) \lesssim 10.8 \%$ [14]. It leads to $\operatorname{Br}\left(H^{-} \rightarrow W+h\right) \times \operatorname{Br}(W \rightarrow \nu+e) \lesssim 0.3 \%$. In case of Fig. 3(a), therefore, the background from $H^{-} \rightarrow W+h$ is much suppressed. In case of $H^{-} \rightarrow \widetilde{\chi}^{-}+\tilde{\chi}^{0}$, the branching ratio is $\operatorname{Br}\left(H^{-} \rightarrow \widetilde{\chi}^{-}+\widetilde{\chi}^{0}\right)$ is about $1 \%$ and $\operatorname{Br}\left(\widetilde{\chi}^{-} \rightarrow e+\widetilde{\nu}\right)$ is roughly $30 \%$ per each lepton flavor. Thus $\operatorname{Br}\left(H^{-} \rightarrow \widetilde{\chi}^{-}+\widetilde{\chi}^{0}\right) \times \operatorname{Br}\left(\widetilde{\chi}^{-} \rightarrow e+\widetilde{\nu}\right)$ is about $0.3 \%$.

As shown in Fig. 3(b), however, if the lighter chargino is dominantly gaugino, the branching ratio of the chargino-neutralino mode increases, so that the branching ratio of $H^{-} \rightarrow \widetilde{\nu}_{1}+\widetilde{e}_{L}$ is relatively decreased. In this case we estimate the probability that the electron is found in the $\tilde{\chi}^{-}+\widetilde{\chi}^{0}$ mode of the charged Higgs decay as $\operatorname{Br}\left(H^{-} \rightarrow\right.$ $\left.\tilde{\chi}^{-}+\widetilde{\chi}^{0}\right) \times \operatorname{Br}\left(\widetilde{\chi}^{-} \rightarrow e+\widetilde{\nu}\right) \simeq 10 \%$. This competes with the probability that an electron is coming out from the $\widetilde{e}_{L}+\widetilde{\nu}_{1}$ decay. We conclude that, even in our specific choice of parameter set, the $\tilde{\chi}^{-}+\widetilde{\chi}^{0}$ mode could be a serious background to search the decay $H^{-} \rightarrow \widetilde{\nu}_{1}+\widetilde{e}_{L}$ when the chargino and neutralino are almost gauginos.

We would like to discuss the testability of the scenario of light $\widetilde{\nu}_{R}$ with unsuppressed $A_{\nu}$ at future collider experiments using the decay $H^{-} \rightarrow \widetilde{\nu}_{1}+\widetilde{e}_{L} \rightarrow e+E_{T}$. An important point is to identify that the observed electron comes from $H^{-}$. It could be achieved using the pair production of the charged Higgs bosons. In a pair production of the charged Higgs, one of the charged Higgs bosons can be identified using the $t+b$ mode. Then if an electron is observed in the charged Higgs pair production it must be identified as one from the decay of another charged Higgs through $H^{-} \rightarrow \widetilde{\nu}_{1}+\widetilde{e}_{L}$. For example, at the $e^{+} e^{-}$linear collider (ILC), the typical size of the cross section of the charged Higgs boson 

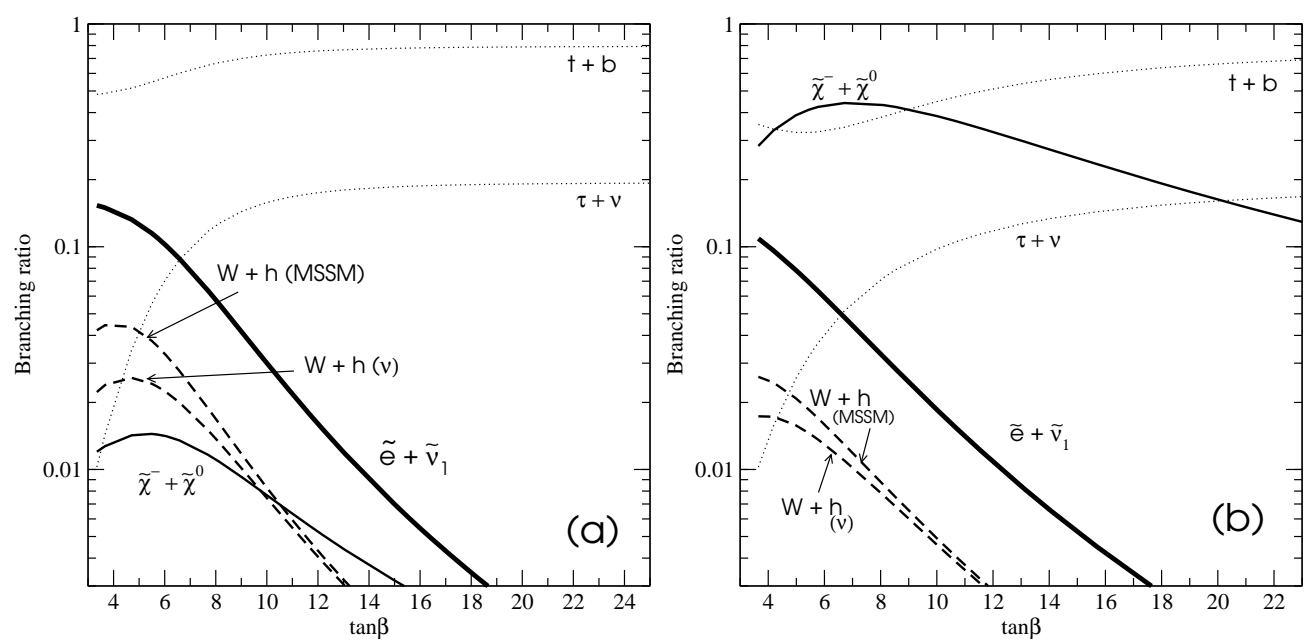

Figure 3: The branching ratios of charged Higgs boson decay for $m_{H^{-}}=350 \mathrm{GeV}$. The decay mode into sneutrino and selectron is found for $m_{\tilde{e}_{L}}=200 \mathrm{GeV}, m_{\tilde{\nu}_{1}}=50 \mathrm{GeV}, A_{\nu}=$ $500 \mathrm{GeV}$. The chargino-neutralino mode is obtained for $m_{\tilde{\chi}_{1}^{-}}=150 \mathrm{GeV}$ with $M_{2} / \mu=5$ (a) and 1 (b).

pair is $O(1-10)(\mathrm{fb})$ for $m_{H^{-}}=O(100 \mathrm{GeV})$ [12]. Assuming the integrated luminosity as $100 \mathrm{fb}^{-1}$, it is expected that $100 \sim 1000$ charged Higgs pairs are produced in a year. Fig. 3(a) tells us that, when $\tan \beta=3$, only few electrons appear from 1000 charged Higgs bosons in the MSSM (the $W+h$ mode), while about 160 electrons from the $\widetilde{e}+\widetilde{\nu}_{1}$ mode is expected in our scenario. Therefore, an excess of electrons from the charged Higgs decay could be a signal of the TeV scale right-handed sneutrino with unsuppressed trilinear coupling $A_{\nu}$.

\section{Summary}

In this paper, we have studied phenomenology of the scenario of $\mathrm{TeV}$ scale right-handed sneutrino inspired by models of SUSY breaking inspired neutrino mass [4, 5, 6]. The important prediction of this scenario is that the sneutrino trilinear coupling $A_{\nu}$ could be sizable and is not suppressed by the neutrino Yukawa coupling. We examined two phenomenological consequences of this scenario. We found that the sneutrino contribution to the lightest Higgs boson mass is destructively interferes with the ordinary MSSM contributions. Thus the lightest Higgs boson mass may be lowered in this model via sneutrino exchange with large $A_{\nu}$. The large $A_{\nu}$ also affects the decay of charged Higgs boson. It is shown that the process $H^{-} \rightarrow \widetilde{\nu}_{1}+\widetilde{e}_{L}$ could be subdominant decay mode in some parameter region and the branching ratio is roughly $\sim 10 \%$ for small $\tan \beta$. In 
such parameter region, we expect that roughly 200 electrons per year from the charged Higgs decay at the ILC experiments with the integrated luminosity $100 \mathrm{fb}^{-1}$. On the other hand the MSSM predicts only few electrons from the charged Higgs decay. The excess of the electrons in the charged Higgs decay, therefore, could be a signal of the $\mathrm{TeV} \widetilde{\nu}_{R}$ scenario.

\section{Acknowledgments}

The work of G.C.C. was supported in part by the Grant-in-Aid for Science Research, Ministry of Education, Science and Culture, Japan (No.K175402386). The work of S.K. was supported by the Japan Society of Promotion of Science.

\section{References}

[1] P. Minkowski, Phys. Lett. B 67, 421 (1977); T. Yanagida, in Proceedings of the workshop on unified theory and baryon number in the universe, O. Sawata and A. Sugamoto eds., KEK report 79-18, Tsukuba, Japan 1979; M. Gell-Mann, P. Ramond and R. Slansky, Complex spinors and unified theories, in Supergravity, D.Z. Freedman and F. van Nieuwenhuizen eds., North Holland, Amsterdam 1979.

[2] M. Fukugita and T. Yanagida, Phys. Lett. B 174, 45 (1986).

[3] M. R. Buckley and H. Murayama, arXiv:hep-ph/0606088.

[4] N. Arkani-Hamed, L. Hall, H. Murayama, D. Smith and N. Weiner, Phys. Rev. D64, 115011, 2001.

[5] F. Borzumati and Y. Nomura, Phys. Rev. D 64, 053005 (2001).

[6] J. March-Russell and S. M. West, Phys. Lett. B 593, 181 (2004).

[7] G. F. Giudice and A. Masiero, Phys. Lett. B 206, 480 (1988).

[8] F. Borzumati, K. Hamaguchi, Y. Nomura and T. Yanagida, hep-ph/0012118.

[9] Y. Okada, M. Yamaguchi and T. Yanagida, Prog. Theor. Phys. 85, 1 (1991).

[10] Y. Okada, M. Yamaguchi and T. Yanagida, Phys. Lett. B 262, 54 (1991).

[11] J. R. Ellis, G. Ridolfi and F. Zwirner, Phys. Lett. B 257, 83 (1991); ibid. Phys. Lett. B 262, 477 (1991); H. E. Haber and R. Hempfling, Phys. Rev. Lett. 66, 1815 (1991). 
[12] A. Djouadi, hep-ph/0503173.

[13] H. E. Haber, R. Hempfling and A. H. Hoang, Z. Phys. C 75, 539 (1997).

[14] W. M. Yao et al. [Particle Data Group], J. Phys. G 33, 1 (2006). 